\title{
The miR-1204 regulates apoptosis in NSCLC cells by targeting DEK
}

\author{
Zhen Qian, Juan Yang, Huanhuan Liu, Yue Yin, Lingling Hou, Honggang Hu
}

College of Life Sciences and Bio-engineering, Beijing Jiaotong University, Beijing 100044, China

\begin{abstract}
Introduction. This study endeavors to analyze the effects of miR-1204 on the expression of DEK oncogene in non-small cell lung cancer (NSCLC) cell lines and to study the molecular mechanisms of these effects.

Material and methods. The miR-1204 mimics and inhibitors were transfected into the (A549 and SPC) NSCLC cells. Then the mRNA levels, cell viability, apoptosis rate, morphology and caspase activity were determined. The expression of apoptosis-related proteins Bcl-2 and Bax was also analyzed.

Results. In NSCLC cell lines (A549 and SPC), DEK mRNA levels were down-regulated in miR-1204 overexpression group. In miR-1204 inhibition group, the expression of DEK mRNA showed an opposite trend. The overexpression of miR-1204 increases the apoptosis rate in NSCLC cells. The Bcl-2 level in the miR-1204 overexpression group was decreased, while the Bax level was increased. In the miR-1204 inhibition group, expression of Bcl-2 and Bax showed opposite trends. Cell staining revealed cell's morphological changes; the apoptosis in the miR-1204 overexpression group revealed significant morphological features, such as brighter nuclei and nuclear condensation. Results indicated a typical characteristic of apoptosis in the miR-1204 overexpression group. Caspase- 9 and Caspase- 3 were involved in the apoptosis pathway, which was mediated by miR-1204 and DEK. Conclusions. The miR-1204 induces apoptosis of NSCLC cells by inhibiting the expression of DEK. The mechanism of apoptosis involves down-regulation of Bcl-2 and up-regulation of Bax expression. Moreover, the apoptosis was mediated by mitochondria-related caspase 9/3 pathway. (Folia Histochemica et Cytobiologica 2019, Vol. 57, No. 2, 64-73)
\end{abstract}

Key words: NSCLC; A549 cells; SPC cells; DEK; miR-1204; apoptosis; Bcl-2; Bax; caspase-3; caspase-9

\section{Introduction}

Lung cancer is the leading cause of cancer-related death worldwide. More than one million cases are diagnosed each year [1]. Lung cancer is morphologically divided into non-small cell lung cancer (NSCLC) and small cell lung cancer, of which NSCLC accounts for about $80 \%$ of all lung cancer cases [2]. Despite the growing number of NSCLC genomics studies and targeted therapies, the overall 5-year survival rate is only $15 \%[3,4]$. This highlights the need for a better understanding of NSCLC biology to improve the prevention, diagnosis and treatment of this cancer [5]. Recently, there is evidence that microRNAs

Correspondence address: Honggang $\mathrm{Hu}$

College of Life Sciences and Bio-engineering,

Beijing Jiaotong University, Beijing, China

e-mail: hghu@bjtu.edu.cn
(miRNAs) may be involved in the pathogenesis of NSCLC, providing new insights into disease biology.

DEK oncogene (DEK) is a ubiquitous protein in multicellular organisms and some single-celled organisms. DEK can promote the repair of DNA damage while also participates in apoptosis [6]. DEK has been already identified as an oncogene and is overexpressed in various malignant tumors, such as colorectal, gastric, neuroendocrine, prostate, breast, and bladder cancer [7-11]. Recent studies indicate that DEK depletion induced astrocytic tumor cell apoptosis with down-regulated expression of Bcl-2 and C-myc; the caspase-3 activity in the astrocytic tumor was also significantly enhanced after the knockdown [12]. Data indicate that DEK plays multiple roles such as promotion of proliferation and migration of cancer cells and facilitation of tumor growth and its maintenance [13]. It can be used as a potential target for astrocytic tumor diagnosis and gene therapy [14]. 
DEK overexpression is an important independent risk factor for overall survival in patients with NSCLC. DEK may potentially be used as an independent biomarker for NSCLC prognosis assessment [15].

MicroRNAs (miRNAs) are a class of endogenous non-coding RNAs of approximately 20-24 nucleotides. MiRNAs play important roles in a variety of cellular processes including proliferation, apoptosis, differentiation, and malignant transformation [13-18]. New evidence has revealed that miRNAs influence the initiation and progression of various malignancies, including lung cancer [19-21]. For example, it is reported that miR-145, miR-21, miR-214, and miR-29c have a crucial role in lung cancer [22-25]. miR-1204 is a member of the PVT1 region. Recent studies have found that miR-1204 may improve B cell differentiation and metastasis in breast cancer and can target PITX1 in NSCLC, which may be a poor prognostic factor and a potential therapeutic target for NSCLC [26].

The aim of this study is to find out if miR-1204 targeting DEK in A549 and SPC cells affects cell apoptosis and apoptosis-related pathways by transfecting the cells with miR-1204 or miR-1204 inhibitors. To examine the potential molecular mechanisms of miR-1204 on apoptosis, we assayed the expression of apoptosis-related proteins (Bcl-2 and Bax), activity of caspase-3, caspase-8, and caspase-9, cell morphology and other biological behaviors of NSCLC cells and explored their related mechanisms.

\section{Materials and methods}

Cell cultures. The A549 and SPC cell lines of NSCLC were purchased from the American Type Culture Collection (Manassas, VA, USA). The cells were maintained in the Dulbecco's Modified Eagle Medium (DMEM) with $10 \%$ fetal bovine serum (FBS), $100 \mathrm{U} / \mathrm{ml}$ penicillin, and $100 \mathrm{mg} / \mathrm{ml}$ streptomycin in humidified air with $5 \% \mathrm{CO}_{2}$ at $37^{\circ} \mathrm{C}$.

miRNA target gene prediction databases. Three miRNA target gene prediction databases were used in research. miRanda: http://www.microrna.org/; TargetScan: http://www. targetscan.org/; miRWalk:http://zmf.umm.uni-heidelberg.de/

The miRNA mimics, siRNAs, cell transfection, and luciferase reporter assays. The $\mathrm{miR}-1204 \mathrm{mimic}, \mathrm{miR}-1204$ inhibitor, control miRNA, DEK siRNA (si-DEK), and control siRNA (si-Control) were obtained from RiboBio (Guangzhou, China). The A549 and SPC cells were transfected with miR-1204 mimics, miR-1204 inhibitors, control miRNA, DEK siRNA, and control siRNA (50 nM) using riboFECT ${ }^{\mathrm{TM}} \mathrm{CP}$ transfection (RiboBio) following the manufacturer's instructions.
The 3'-UTR of the DEK gene containing the putative binding sites for miR-1204 was infused into a pGL vector purchased from Hai Chuang Biological Technology Co. (Beijing, China). Cells were calibrated into 24-well plates and cotransfected with miR-1204 mimics, miR-1204 inhibitors, or control miRNA with $0.8 \mu \mathrm{g}$ luciferase reporter plasmids, or control reporter vectors. Luciferase activity was measured after $48 \mathrm{~h}$ of transfection using a dual-luciferase assay system (\#E2920; Promega, Madison, WI, USA). Results were normalized to Renilla luciferase activity.

Real-time RT-PCR. Total RNA was extracted from NSCLC cells using Trizol reagent (Invitrogen, Carlsbad, CA, USA). The expression of miR-1204 was evaluated with miDETECT, a Track TMmiRNA qRT-PCR Starter Kit (RiboBio). Real-time PCR was conducted using the GoTaq Real-Time PCR Systems (Promega) to evaluate the DEK expression. B2M level was used as a control for the real-time PCR analysis. The primers for B2M were: sense, 5'-GGCTATCCAGCGTACTCC-3'; anti-sense, 5'-ACGGCAGGCATACTCATC-3'. Primers for DEK were: sense, 5'-TGTTAAGAAAGCAGATAGCAGCACC-3'; anti-sense 5'-ATTAAAGGTTCATCATCTGAACTATCCTC-3'. The primers for Bcl-2 were: sense, 5'-GGCTATCCAGCGTACTCC-3'; anti-sense 5'-ACGGCAGGCATACTCATC-3'. The primers for Bax were: sense, 5'-GGAGCTGCAGAGGATGATTG-3'; anti-sense, 5'-CCTCCCAGAAAAATGCCATA -3'. The results were analyzed by the following formula: $2^{-\Delta \Delta \mathrm{Ct}}$.

Cell viability assay. The cells were seeded into 96-well plates $\left(3 \times 10^{4}\right.$ cells/well $)$ and evaluated for $10-60 \mathrm{~h}$ after transfection. The process was repeated thrice in each group. The cell viability was determined by the addition of Cell Counting Kit-8 (MedChem Express, Beijing, China). The CCK-8 kit, based on the chemical reactivity of WST-8 (2-(2-methoxy-4-nitro))-3-(4-nitrophenyl)-5-(2,4-disulfophenyl)-2H-tetrazole monosodium salt), is a rapid and highly sensitive kit widely used for the assessment of cell proliferation and cytotoxicity. WST- 8 belongs to the upgraded product of MTT. In the presence of electron coupling reagent WST-8 can be reduced by mitochondrial dehydrogenases to produce highly water-soluble orange-yellow formazan product. Cell proliferation is proportional and inversely proportional to cytotoxicity. The OD value was measured at a wavelength of $450 \mathrm{~mm}$ using a microplate reader (TECAN, Vienna, Austria), indirectly reflecting the number of viable cells.

Analysis of cell apoptosis. Annexin V-FITC/PI kit (Keygen Biotech, Jiangsu, China) was used to assay the apoptosis. The cells were collected after 48 hours of transfection in accordance with the manufacturer's instructions. Later, the cells were incubated with Annexin V - fluorescein isothiocyanate and propidium iodide for $15 \mathrm{~min}$ in the dark at 
DEK 3'-UTR 802-826 5' AGUAUUAACUCUGUaaaagugug ( | | | | || || hsa-miR-1204 3' AUAAGGGAGACCAaggccacga

Figure 1. Complementarity site for miR-1204 in the 3'-UTR region of DEK

$25^{\circ} \mathrm{C}$ to analyze the percentage of apoptotic cells. Cells were analyzed with a FACS Calibur flow cytometer (NovoCyte, Beijing, China).

Assessment of cell morphology. The cells were assayed after $48 \mathrm{~h}$ of transfection and stained with Hoechst $33258(10 \mathrm{mg} / \mathrm{ml})$ for $20 \mathrm{~min}$ at $37^{\circ} \mathrm{C}$. Later, after washing the cells with phosphate-buffered saline (PBS), the morphology of the cells was monitored and photographed under TE-2000S fluorescence microscope (Nikon, Tokyo, Japan).

Caspase activity assay. The cells $(2 \times)$ were seeded in 6 -well plates and collected $48 \mathrm{~h}$ after transfection. The cells were treated according to the protocol described, with Caspase-3, -8, and -9 Fluorescence Assay Kits (Biovision, San Francisco, CA, USA). The activities of caspase-3, -8, and -9 were measured using a fluorescence spectrophotometer (BMG LABTECH, Baden, Stuttgart, Germany) with an excitation wavelength of $400 \mathrm{~nm}$ and emission wavelength of $505 \mathrm{~nm}$.

Statistical analysis. Statistical analysis was carried out using the SPSS version 2.0 (IBM, Armonk, NY, USA). Differences between two groups were enumerated using the two-tailed Student's t-tests. $P<0.05$ was considered to illustrate a statistically significant difference, and all values were expressed as mean \pm SEM.

\section{Results}

\section{The prediction of DEK as a target of miR-1204}

Earlier results from the sequencing analysis had indicated that there were large numbers of differentially expressed sequences between the DEK silencing group and the DEK silencing control group in A549 lung cancer cells, especially the miRNA sequences. Hence, it was reasonably presumed that DEK might be regulated by miRNA.

Three miRNA target gene prediction databases (miRanda, TargetScan, and miRWalk) were applied to predict the potential binding sites of miR-1204, where DEK was the target gene (Fig. 1).

\section{The miR-1204 overexpression and inhibition}

The study was successful in detecting the expression level of miR-1204, 48 hours after transfection with
miR-1204 mimic, inhibitor, or corresponding control respectively in A549 or SPC cells. The level of expression of miR-1204 in the miR-1204 mimic group was significantly higher than that in the control group, while the expression level of miR-1204 in miR-1204 inhibitor group was apparently lower than that in the control group (Fig. 2A).

\section{The miR-1204 inhibits the activity of DEK 3'-UTR}

DEK 3'-UTR luciferase reporter plasmid was co-transfected with miR-1204 mimic, inhibitor, or corresponding control, respectively, into A549 or SPC cells. After transfection for 48 hours, the cells were collected and the luciferase activity was measured. The luciferase activity of A549 and SPC cells transfected with DEK 3' -UTR encoding plasmid was observed to have been decreased in miR-1204 overexpression group. Meanwhile, the luciferase activity with DEK 3'-UTR encoding plasmid was found to have been increased in the miR-1240 inhibition group (Fig. 2B).

\section{The miR-1204 inhibits DEK expression}

The miR-1204 mimic, inhibitor, and the corresponding control were transfected in A549 and SPC cells. Thereafter, the DEK mRNA level was examined through the RT-PCR analysis. The resultant data indicated a decrease of DEK expression in the miR1204 overexpression group when compared with the control group, whereas the DEK expression in the miR-1204 inhibition group indicated an opposite trend (Fig. 2C).

\section{The miR-1204 overexpression increases apoptosis in NSCLC cells}

To substantiate the premise that miR-1204 regulates apoptosis in NSCLC cells, we transfected miR-1204 mimic, inhibitor, or the corresponding control, respectively, into A549 and SPC cells, and measured the apoptosis rate using the flow cytometer. The apoptosis rate in miR-1204 overexpression group was found to have significantly increased, while it had decreased in the miR-1204 inhibition group (Fig. 3A-D).

By using Hoechst 333258 staining technique, we observed the changes in the cell morphology with a fluorescence microscope. The miR-1204 overexpression group revealed characteristic morphological features of apoptosis such as brighter nuclei and nuclear condensation (Fig. 3E-F).

\section{The miR-1204 inhibits proliferation of NSCLC cells}

The CCK- 8 assay showed that miR-1204 inhibited the proliferation of NSCLC cells. We transfected miR-1204 mimic, inhibitor, or corresponding control into A549 and SPC cells, and the cell viability was 


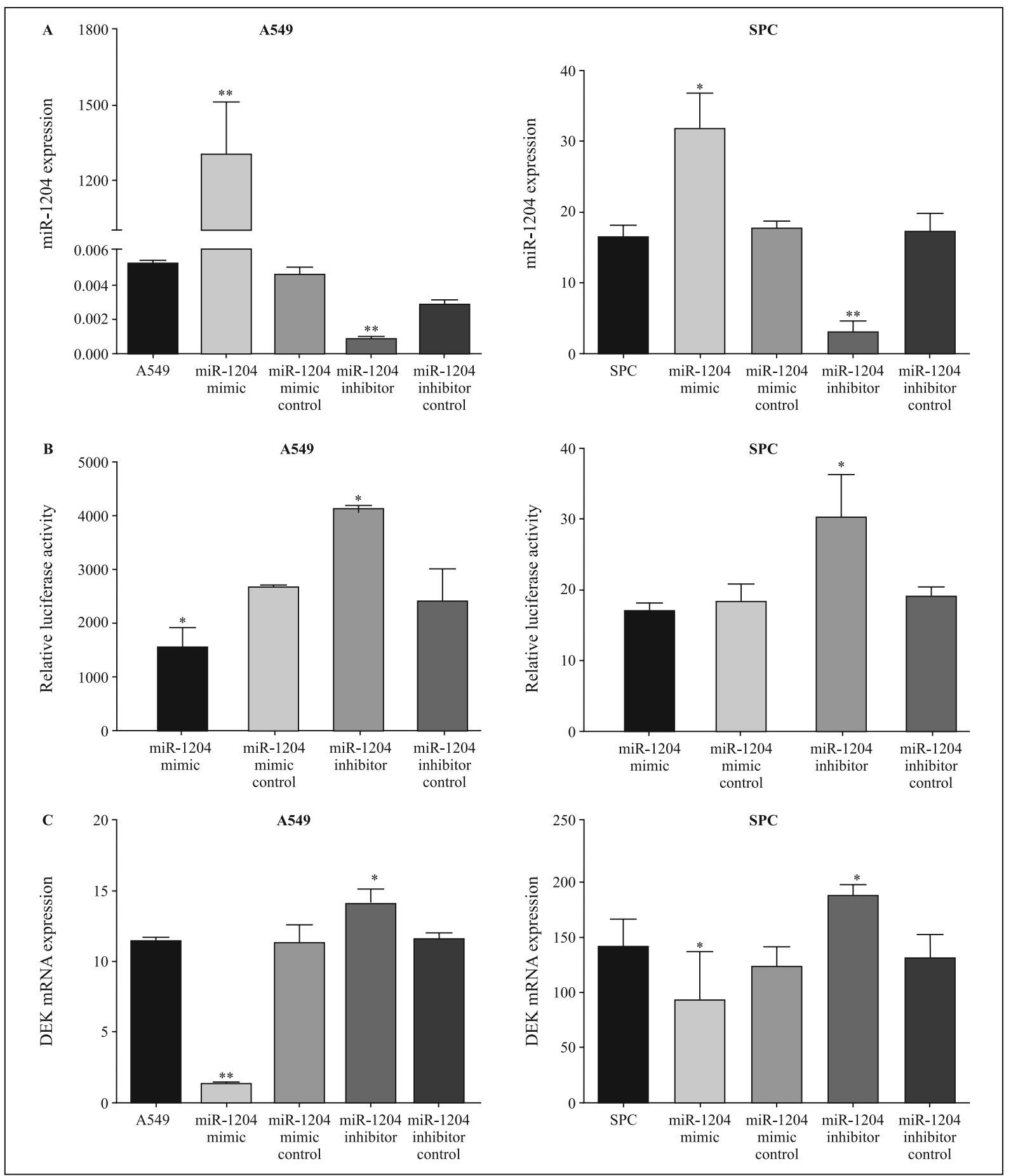

Figure 2. miR-1204 overexpression and inhibition in A549 and SPC cells. A. Real-time RT-PCR detection of miR-1204 expression in A549 and SPC cells transfected with miR-1204 mimic (mimic, inhibitor or corresponding controls). B. Luciferase activity in A549 and SPC cells transfected with DEK 3'-UTR luciferase reporter plasmid and miR-1204 mimic (mimic, inhibitor or corresponding controls). C. Detection of DEK mRNA expression in A549 and SPC cells transfected with miR-1204 mimic (mimic, inhibitor or corresponding controls) by real-time RT-PCR. ${ }^{*} \mathrm{P}<0.05$, ${ }^{*} \mathrm{P}<0.01$ compared with mimic or inhibitor control transfected cells. Data are presented as the mean \pm SEM of three independent experiments.

determined after $10-60 \mathrm{~h}$. It was found that miR-1204 significantly inhibited the proliferation of NSCLC cells and that the inhibitory effect of miR-1204 was time-dependent (Fig. 4).

\section{Effect of siDEK on expression of Bcl-2 and Bax}

We transfected A549 and SPC cells with siDEK or control, and then detected mRNA expression levels of Bcl-2 and Bax. It was demonstrated that Bcl-2 


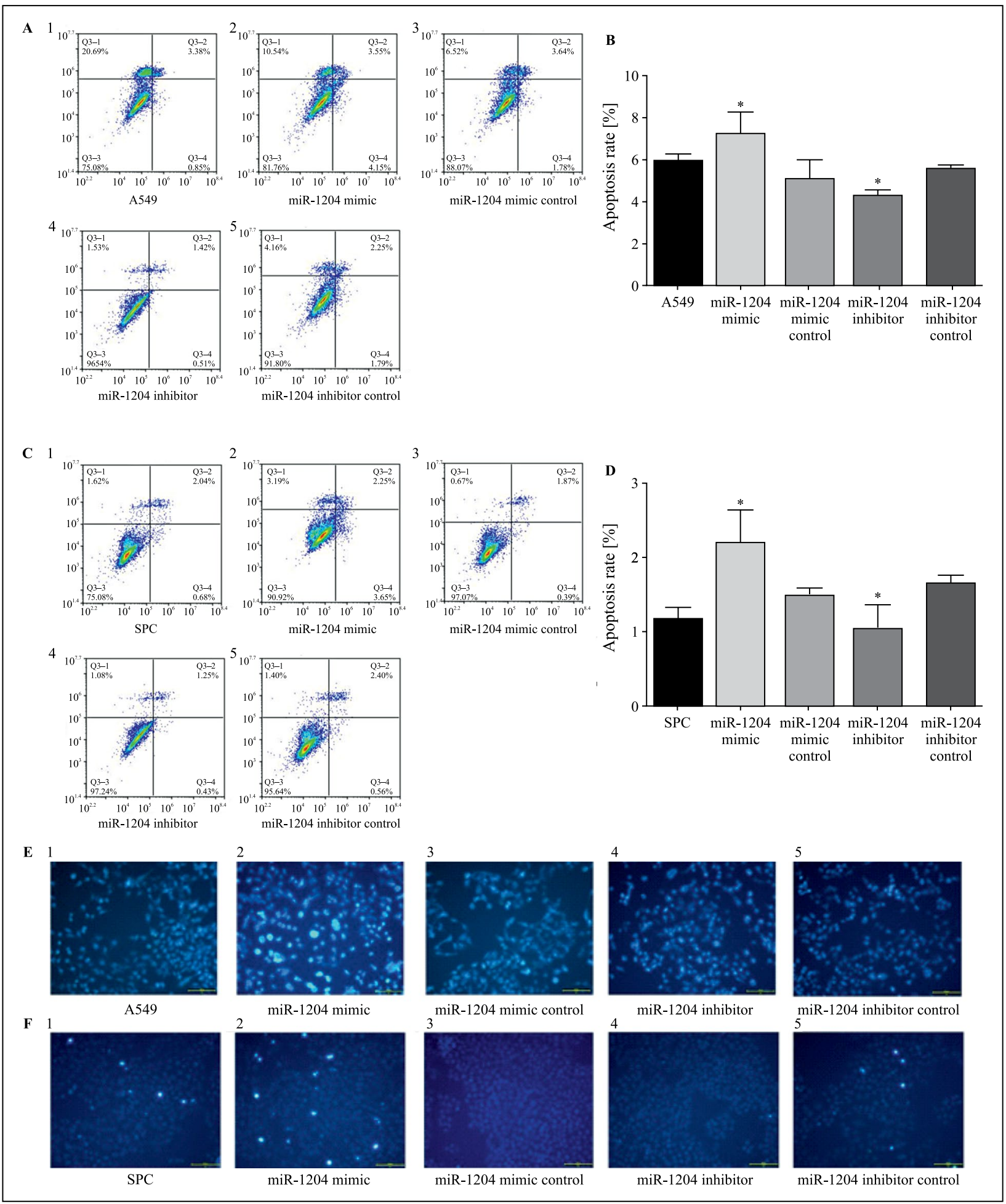

Figure 3. miR-1204 induced apoptosis in A549 and SPC cells. A. A549 cells apoptosis was detected by flow cytometry. Numbers 1-5 represent the A549, miR-1204 mimic, miR-1204 mimic control, miR-1204 inhibitor, miR-1204 inhibitor control. B. Apoptosis rate in A549 cells after transfected with miR-1204 mimic (mimic, inhibitor or corresponding controls). C. SPC cells apoptosis was detected by flow cytometry. Numbers 1-5 represent the SPC, miR-1204 mimic, miR-1204 mimic control, miR-1204 inhibitor, miR-1204 inhibitor control. D. Apoptosis rate in SPC cells after transfected with miR-1204 mimic (mimic, inhibitor or corresponding controls). ${ }^{*} \mathrm{P}<0.05$, ${ }^{*} \mathrm{P}<0.01$ compared with mimic or inhibitor control transfected cells. Data are presented as the mean \pm SEM of three independent experiments. E. Nuclear morphology was observed in A549 cells by Hoechst 33258 staining. Numbers 1-5 represent the A549, miR-1204 mimic, miR-1204 mimic control, miR-1204 inhibitor, miR-1204 inhibitor control (scale bars: $100 \mu \mathrm{m}$ ). F. Nuclear morphology was observed in SPC cells by Hoechst 33258 staining. Numbers 1-5 represent the SPC, miR-1204 mimic, miR-1204 mimic control, miR-1204 inhibitor, miR-1204 inhibitor control. Apoptotic cells with bright, nuclear condensation. Scale bars: $100 \mu \mathrm{m}$. 


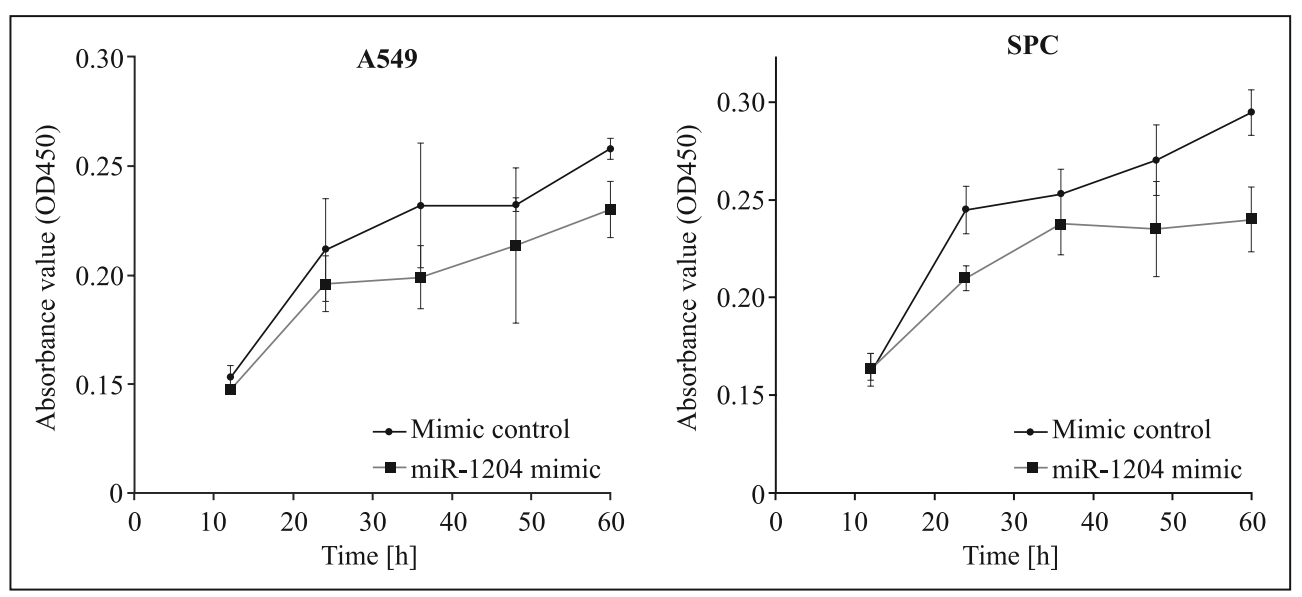

Figure 4. Cell proliferation of A549 and SPC. Cells were transfected with miR-1204 mimic or miR-1204 mimic control and measured by CCK- 8 assays. The absorbance was measured at $450 \mathrm{~nm}$ as described in Methods.

expression was significantly decreased in siDEK group compared with the control group, while Bax expression was significantly increased (Fig. 5A-C).

\section{Effects of miR-1204 on the expression of Bcl-2 and Bax}

The miR-1204 mimic, inhibitor, or corresponding control were transfected into A549 and SPC cells and the mRNA expression levels of Bcl-2 and Bax were determined. The Bcl-2 expression was significantly decreased in the miR-1204 overexpression group compared with the control group, while the Bax expression was significantly increased. In the miR-1204 inhibition group, the opposite results were observed (Fig. 6A, B).

\section{Activation of caspase-3, caspase-8, and caspase-9 during miR-1204-induced apoptosis}

The activation of caspases in miR-1204 induced cell apoptosis were investigated sequentially. As Figure 7 shows, in the both cell lines, A549 and SPC, caspase-3 activity increased in the miR-1204 overexpression group. Caspase-9 activity was also observed to have increased. However, no change in caspase- 8 activity was detected in any of the cell groups tested (Fig. 7A, B).

\section{Discussion}

miR-1204 is a member of the PVT1 region. Recent studies have found that miR-1204 may improve B cell differentiation and metastasis in breast cancer and can target PITX1 in non-small cell lung cancer, which may be a poor prognostic factor and a potential therapeutic target for NSCLC [26]. We used three miRNA target prediction databases (miRanda, TargetScan, and miRWalk) to determine the target gene of miR1204. It was found that one target gene of miR-1204 was DEK. The DEK overexpression is prevalent in numerous types of malignant cancers [7-8]. Recently, many studies have found DEK to be carcinogenic in lung cancer formation [9-11]. However, there is very little research on the role of microRNA in DEK. Hence, we proposed the hypothesis that the expression of DEK could be regulated by miR-1204. With an intent to confirm this hypothesis, first, we used luciferase reporter assay to confirm DEK as a target gene of miR-1204. Moreover, we also found that the mRNA levels of DEK were negatively correlated with the expression of miR-1204. The above results provided evidence to substantiate the hypothesis that the expression of DEK in NSCLC was negatively regulated by miR-1204.

Next, to reveal the effects of the miR-1204 on NSCLC cells by regulating DEK, the effect of miR1204 on apoptosis of A549 and SPC cells were investigated. The apoptosis is a programmed cell death and an important physiological process to maintain homeostasis [27]. Alterations of the apoptosis play a significant role in the survival of abnormal cells and tumorigenesis [28, 29]. Cell apoptosis is characterized by chromatin concentration, DNA fragmentation, membrane foaming, and/or apoptotic bodies [25-30]. In our study, Annexin V FITC analysis of flow cytometry and in vitro Hochest 33258 staining analysis indicated that the apoptosis rate of A549 and SPC cells in miR-1204 overexpression group was increased, while the apoptosis rate was found to have decreased in the miR-1204 inhibited group. The cell viability of the miR-1204 overexpression group was significantly lower than that of the control group, whereas, the 


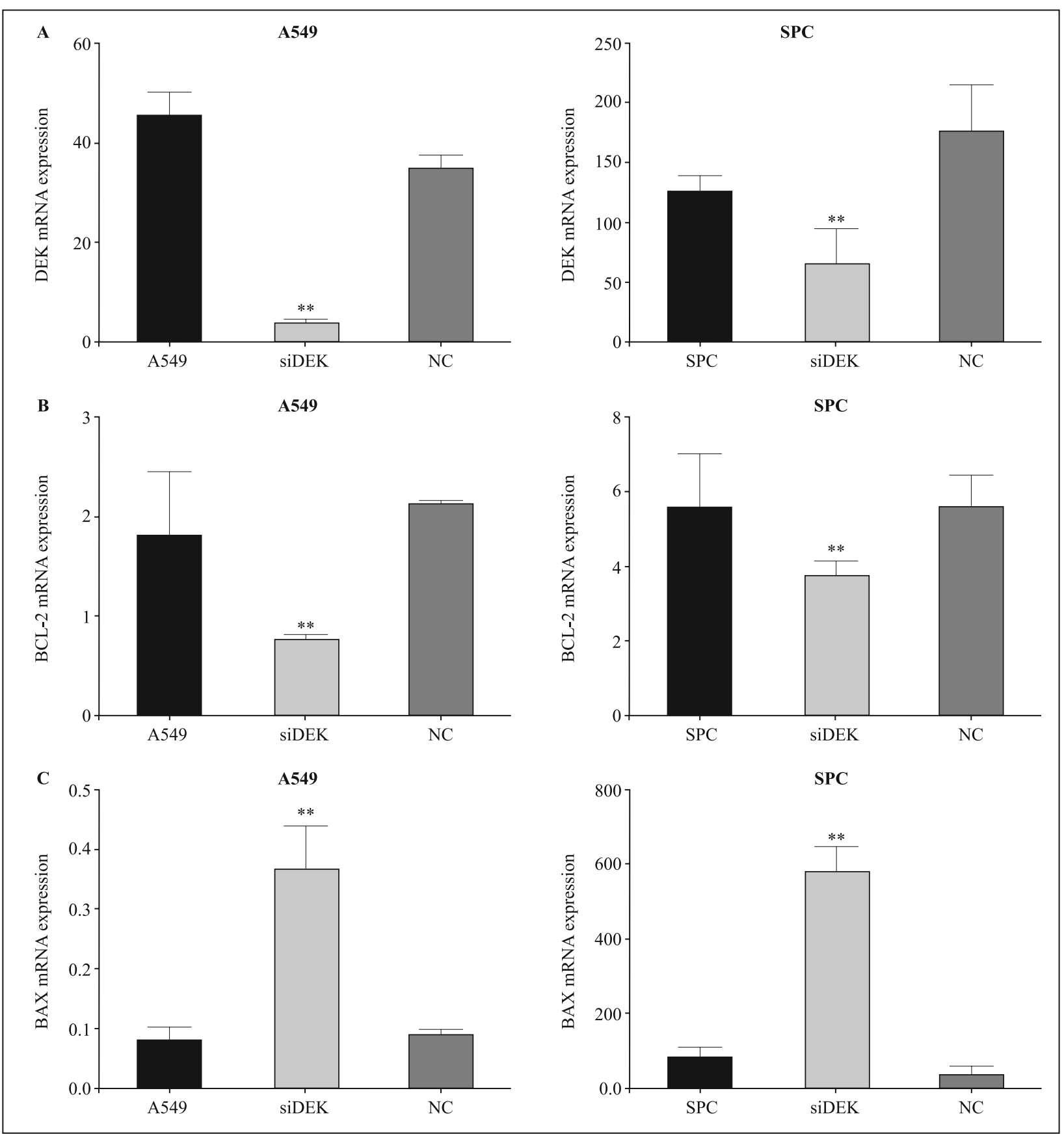

Figure 5. Expression of Bcl-2 and Bax affected by siRNA DEK. A. Real-time RT-PCR detection of DEK expression in A549 and SPC cells transfected with siDEK or controls. B. Real-time RT-PCR detection of Bcl-2 expression in A549 and SPC cells transfected with siDEK or controls. C. Real-time RT-PCR detection of Bax expression in A549 and SPC cells transfected with siDEK or controls. ${ }^{*} \mathrm{P}<0.05,{ }^{*} \mathrm{P}<0.01$ compared with control group of transfected cells. Data are presented as the mean \pm SEM of three independent experiments.

miR-1204 inhibited group expressed an opposite trend. These results evidenced that miR-1204 overexpression induced apoptosis in A549 and SPC cells and inhibited their growth.

Apoptosis is strictly regulated by the multiple pathways and proteins. The members of the Bcl-2 family play a crucial role in inhibiting or promoting apoptosis [31]. The Bcl-2 family consists of anti-apoptotic (Bcl-2, Bcl-w, Bcl-xl, A1) and pro-apoptotic molecules (Bax, Bcl-xs, Bad, Bak, Bid, Bim, Bik) [12, 31]. Bax protein allows some ions and small molecules such as cytochrome $\mathrm{C}$ to penetrate the mitochondrial 


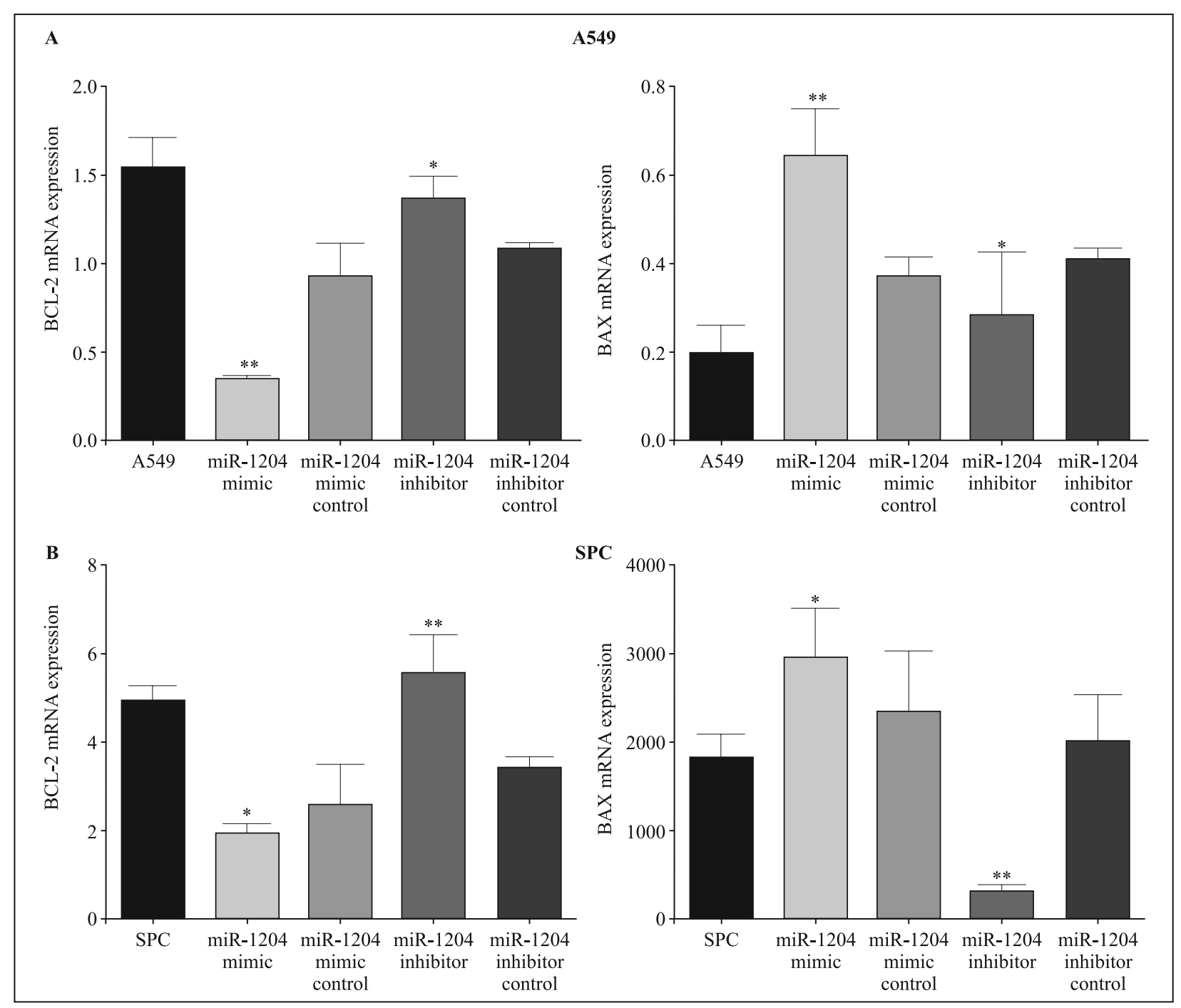

Figure 6. Expression of Bcl-2 and Bax affected by miR-1204. A. Bcl-2 and Bax mRNA expression in A549 cells transfected with miR-1204 mimic (mimic, inhibitor or corresponding controls) was detected by real-time PCR. B. Bcl-2 and Bax mRNA expression in SPC cells transfected with miR-1204 mimic (mimic, inhibitor or corresponding controls) was detected by real-time RT-PCR. Data are presented as the mean \pm SEM of three independent experiments.

membrane and enter the cytoplasm, leading to apoptosis. However, $\mathrm{Bcl}-2$ has the opposite function. It can counteract the pore-forming activity of Bax and prevent the free penetration of small molecules, thus protecting the cells from imminent apoptosis [12]. This study detected the decreased $\mathrm{Bcl}-2$ expression and the increased Bax expression in NSCLC cells with miR-1204 overexpression, while the miR-1204 inhibition showed opposite results in the cells. Hence, we presume that the $\mathrm{Bcl}-2$ and $\mathrm{Bax}$ proteins were involved in the miR-1204-induced apoptosis of A549 and SPC cells.

Caspases are intracellular cysteine protease, which are capable of inducing apoptosis through two major pathways, namely: (i) FADD-caspase-8/3 and (ii) cytochrome C (CytC)-caspase-9/3 [29].The FADD-caspase-8/3 pathway is triggered by Fas/ /FasL, while the cytc-caspase-9/3 pathway is triggered by mitochondrial damage. Later, caspase-3 (an endogenous endonuclease) is activated, leading to the irreversible apoptosis [32,33]. The activity changes of caspase- 3 , caspase- 8 , and caspase- 9 by colorimetric assay in increased apoptosis group were determined. The results indicated that the activity of caspase- 3 and caspase- 9 were significantly increased, while the activity of caspase- 8 was not affected by miR-1204. Thus, our results suggest that the main mechanism by which miR-1204 induced cell apoptosis acted via 


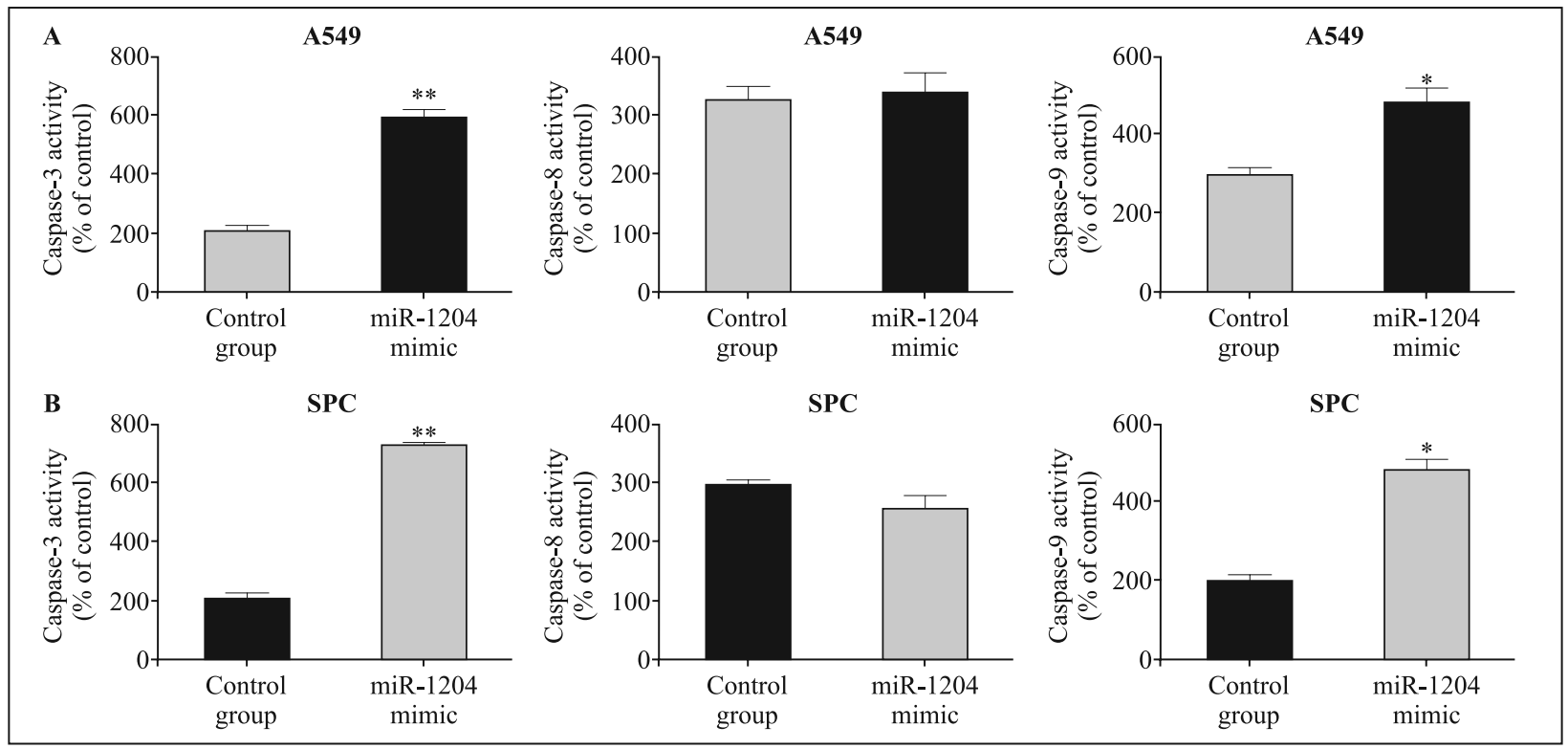

Figure 7. Caspase-3, -8 and -9 activity. Caspases' activities were detected in A549 cells (A) and SPC cells (B). ${ }^{*} \mathrm{P}<0.05$, $* * \mathrm{P}<0.01$ compared with control group of transfected cells. Data are presented as the mean \pm SEM of three independent experiments.

mitochondria-related CytC-caspase-9/3 pathway and not the caspase- 8 pathway.

In summary, there are only a few studies related to the role of miRNA in NSCLC, while a better understanding of miRNA-mediated gene expression regulation is needed. Our study is the first to indicate that DEK is negatively regulated by miR-1204 in two NSCLC cell lines and that miR-1204 could promote apoptosis of the NSCLC cells by inhibiting the expression of DEK. The mechanism of miR-1204 induced apoptosis involves down-regulation of $\mathrm{Bcl}-2$ and up-regulation of Bax expression. Besides, this apoptosis is mediated by mitochondria related caspase 9/3 pathway. However, further in-depth research is needed to elucidate the precise mechanisms of these effects.

\section{References}

1. Spiro SG, Silvestri GA. One hundred years of lung cancer. Am J Respir Crit Care Med. 2005; 172(5): 523-529, doi: 10.1164/ rccm.200504-531OE, indexed in Pubmed: 15961694.

2. Katanoda K, Yako-Suketomo H. Trends in lung cancer mortality rates in Japan, USA, UK, France and Korea based on the WHO mortality database. Jpn J Clin Oncol. 2012; 42(3): 239_ -240, doi: 10.1093/jjco/hys021, indexed in Pubmed: 22375029.

3. Guttmann DM, Kobie J, Grover S, et al. National disparities in treatment package time for resected locally advanced head and neck cancer and impact on overall survival. Head Neck. 2018; 40(6): 1147-1155, doi: 10.1002/hed.25091, indexed in Pubmed: 29394465.

4. Fitzmaurice C, Allen C, Barber RM, et al. Global Burden of Disease Cancer Collaboration. Global, Regional, and National Cancer Incidence, Mortality, Years of Life Lost, Years
Lived With Disability, and Disability-Adjusted Life-years for 32 Cancer Groups, 1990 to 2015: A Systematic Analysis for the Global Burden of Disease Study. JAMA Oncol. 2017; 3(4): 524-548, doi: 10.1001/jamaoncol.2016.5688, indexed in Pubmed: 27918777.

5. Han R, Zheng R, Zhang S, et al. [Trend analyses on the differences of lung cancer incidence between gender, area and average age in China during 1989-2008]. Zhongguo Fei Ai Za Zhi. 2013; 16(9): 445-451, doi: 10.3779/j.issn.10093419.2013.09.02, indexed in Pubmed: 24034990.

6. Alexiadis V, Waldmann $\mathrm{T}$, Andersen $\mathrm{J}$, et al. The protein encoded by the proto-oncogene DEK changes the topology of chromatin and reduces the efficiency of DNA replication in a chromatin-specific manner. Genes Dev. 2000; 14(11): 1308-1312, indexed in Pubmed: 10837023.

7. Lin L, Piao J, Gao W, et al. DEK over expression as an independent biomarker for poor prognosis in colorectal cancer. BMC Cancer. 2013; 13: 366, doi: 10.1186/1471-2407-13-366, indexed in Pubmed: 23902796.

8. Piao J, Shang Y, Liu S, et al. High expression of DEK predicts poor prognosis of gastric adenocarcinoma. Diagn Pathol. 2014; 9: 67, doi: 10.1186/1746-1596-9-67, indexed in Pubmed: 24650035.

9. Lin D, Dong X, Wang K, et al. Identification of DEK as a potential therapeutic target for neuroendocrine prostate cancer. Oncotarget. 2015; 6(3): 1806-1820, doi: 10.18632/ oncotarget.2809, indexed in Pubmed: 25544761.

10. Liu S, Wang X, Sun F, et al. DEK overexpression is correlated with the clinical features of breast cancer. Pathol Int. 2012; 62(3): 176-181, doi: 10.1111/j.1440-1827.2011.02775.x, indexed in Pubmed: 22360505.

11. Datta A, Adelson ME, Mogilevkin Y, et al. Oncoprotein DEK as a tissue and urinary biomarker for bladder cancer. BMC Cancer. 2011; 11: 234, doi: 10.1186/1471-2407-11-234, indexed in Pubmed: 21663673.

12. Ashkenazi A, Fairbrother WJ, Leverson JD, et al. From basic apoptosis discoveries to advanced selective BCL-2 family 
inhibitors. Nat Rev Drug Discov. 2017; 16(4): 273-284, doi: 10.1038/nrd.2016.253, indexed in Pubmed: 28209992.

13. Yu Le, Huang X, Zhang W, et al. Critical role of DEK and its regulation in tumorigenesis and metastasis of hepatocellular carcinoma. Oncotarget. 2016; 7(18): 26844-26855, doi: 10.18632/oncotarget.8565, indexed in Pubmed: 27057626.

14. Feng T, Liu Y, Li C, et al. DEK proto-oncogene is highly expressed in astrocytic tumors and regulates glioblastoma cell proliferation and apoptosis. Tumour Biol. 2017; 39(7): 1010428317716248, doi: $10.1177 / 1010428317716248$, indexed in Pubmed: 28670979.

15. Liu X, Qi D, Qi J, et al. Significance of DEK overexpression for the prognostic evaluation of non-small cell lung carcinoma. Oncol Rep. 2016; 35(1): 155-162, doi: 10.3892/or.2015.4365, indexed in Pubmed: 26530274.

16. Liu Y, Han Y, Zhang Hu, et al. Synthetic miRNA-mowers targeting miR-183-96-182 cluster or miR-210 inhibit growth and migration and induce apoptosis in bladder cancer cells. PLoS One. 2012; 7(12): e52280, doi: 10.1371/journal.pone.0052280, indexed in Pubmed: 23284967.

17. Peck BC, Sincavage J, Feinstein S, et al. miR-30 Family Controls Proliferation and Differentiation of Intestinal Epithelial Cell Models by Directing a Broad Gene Expression Program That Includes SOX9 and the Ubiquitin Ligase Pathway. J Biol Chem. 2016; 291(31): 15975-15984.

18. Nie JH, Chen ZH, Shao CL, et al. Analysis of the miRNA-mRNA networks in malignant transformation BEAS-2B cells induced by alpha-particles. J Toxicol Environ Health A. 2016; 79(9-10): 427-435, doi: 10.1080/15287394.2016.1176628, indexed in Pubmed: 27267825.

19. Chen Y, Zhu X, Zhang X, et al. Nanoparticles modified with tumor-targeting scFv deliver siRNA and miRNA for cancer therapy. Mol Ther. 2010; 18(9): 1650-1656, doi: 10.1038/ mt.2010.136, indexed in Pubmed: 20606648.

20. Wang Z, Liao H, Deng Z, et al. miRNA-205 affects infiltration and metastasis of breast cancer. Biochem Biophys Res Commun. 2013; 441(1): 139-143, doi: 10.1016/j.bbrc.2013.10.025, indexed in Pubmed: 24129185.

21. Yao Yu, Suo AL, Li ZF, et al. MicroRNA profiling of human gastric cancer. Mol Med Rep. 2009; 2(6): 963-970, doi: 10.3892/mmr 00000199, indexed in Pubmed: 21475928.

22. Chen Z, Zeng H, Guo Y, et al. miRNA-145 inhibits non-small cell lung cancer cell proliferation by targeting c-Myc. J Exp Clin Cancer Res. 2010; 29: 151, doi: 10.1186/1756-9966-29-151, indexed in Pubmed: 21092188.
23. Wang ZX, Bian HB, Wang JR, et al. Prognostic significance of serum miRNA-21 expression in human non-small cell lung cancer. J Surg Oncol. 2011; 104(7): 847-851, doi: 10.1002/ jso.22008, indexed in Pubmed: 21721011.

24. Salim H, Akbar NS, Zong D, et al. miRNA-214 modulates radiotherapy response of non-small cell lung cancer cells through regulation of p38MAPK, apoptosis and senescence. Br J Cancer. 2012; 107(8): 1361-1373, doi: 10.1038/ bjc.2012.382, indexed in Pubmed: 22929890.

25. Wang $\mathrm{H}$, Zhu Y, Zhao M, et al. miRNA-29c suppresses lung cancer cell adhesion to extracellular matrix and metastasis by targeting integrin $\beta 1$ and matrix metalloproteinase 2 (MMP2). PLoS One. 2013; 8(8): e70192, doi: 10.1371/journal. pone.0070192, indexed in Pubmed: 23936390.

26. Jiang W, He Y, Shi Y, et al. MicroRNA-1204 promotes cell proliferation by regulating PITX1 in non-small-cell lung cancer. Cell Biol Int. 2019; 43(3): 253-264, doi: 10.1002/ cbin.11083, indexed in Pubmed: 30549141.

27. Danial NN, Korsmeyer SJ. Cell death: critical control points. Cell. 2004; 116(2): 205-219, doi: 10.1016/S00928674(04)00046-7, indexed in Pubmed: 14744432.

28. Youle RJ, Strasser A. The BCL-2 protein family: opposing activities that mediate cell death. Nat Rev Mol Cell Biol. 2008; 9(1): 47-59, doi: 10.1038/nrm2308, indexed in Pubmed: 18097445.

29. Dejean LM, Martinez-Caballero S, Guo L, et al. Oligomeric Bax is a component of the putative cytochrome c release channel MAC, mitochondrial apoptosis-induced channel. Mol Biol Cell. 2005; 16(5): 2424-2432, doi: 10.1091/mbc.e04-121111, indexed in Pubmed: 15772159.

30. Saba JD, Obeid LM, Hannun YA. Ceramide: an intracellular mediator of apoptosis and growth suppression. Philos Trans R Soc Lond B Biol Sci. 1996; 351(1336): 233-40; discussion 240, doi: 10.1098/rstb.1996.0021, indexed in Pubmed: 8650271.

31. García S, Liz M, Gómez-Reino JJ, et al. Akt activity protects rheumatoid synovial fibroblasts from Fas-induced apoptosis by inhibition of Bid cleavage. Arthritis Res Ther. 2010; 12(1): R33, doi: 10.1186/ar2941, indexed in Pubmed: 20187936.

32. Rahman MdA, Kim NH, Huh SO. Cytotoxic effect of gambogic acid on SH-SY5Y neuroblastoma cells is mediated by intrinsic caspase-dependent signaling pathway. Mol Cell Biochem. 2013; 377(1-2): 187-196, doi: 10.1007/s11010-0131584-z, indexed in Pubmed: 23404459.

33. Smith MA, Schnellmann RG. Calpains, mitochondria, and apoptosis. Cardiovasc Res. 2012; 96(1): 32-37, doi: 10.1093/ cvr/cvs163, indexed in Pubmed: 22581845.

Submitted: 31 March, 2019

Accepted after reviews: 17 May, 2019 Available as AoP: 27 June, 2019 